\title{
La précarisation des parcours sur le segment secondaire de l'emploi à la Commission européenne de Bruxelles
}

\author{
Magali Ballatore \& Jean-Baptiste Bertrand
}

Émulations - Revue de sciences sociales

$2018, n^{\circ} 28$, « Précarité, précaires, précariat. Allers-retours internationaux ».

\section{Article disponible à l'adresse suivante}

https://ojs.uclouvain.be/index.php/emulations/article/view/ballatore

\section{Pour citer cet article}

Magali Ballatore, Jean-Baptiste Bertrand, « La précarisation des parcours sur le segment secondaire de l'emploi à la Commission européenne de Bruxelles», Émulations, n² 28 , Mise en ligne le 20 février 2019.

DOI : 10.14428/emulations.028.04

Distribution électronique : Université catholique de Louvain (Belgique) : ojs.uclouvain.be

(c) Cet article est mis à disposition selon les termes de la Licence Creative Commons Attribution, Pas d'Utilisation Commerciale 4.0 International. http://creativecommons.org/licenses/by-nc/4.0/

Éditeur : Émulations - Revue de sciences sociales / Presses universitaires de Louvain https://ojs.uclouvain.be/index.php/emulations

ISSN électronique : 1784-5734

PUL PRESSES UNIVERSITAIRES 


\title{
La précarisation des parcours sur le segment secondaire de l'emploi à la Commission européenne de Bruxelles
}

\author{
Magali Ballatore ${ }^{1}$, Jean-Baptiste Bertrand ${ }^{2}$
}

\begin{abstract}
[Résumé] La Commission européenne (CE) à Bruxelles est souvent vue comme un corps de fonctionnaires privilégiés à la fibre européenne. Cependant, au cœur de cette institution aujourd'hui, existent différents types de travailleurs sous des statuts professionnels variés. Regarder de plus près la morphologie sociale et la diversité des situations que connaissent ces travailleurs périphériques, ainsi que le phénomène d'hybridation au sein de la $C E$, fait apparaît assez clairement un certain fossé entre la position de permanents et de nonpermanents. Dans cet article nous nous intéressons à cette population de travailleurs qualifiés en emploi temporaire. Ce faisant, nous essayons de dresser un portrait du passé migratoire, scolaire et/ou professionnel, ainsi que des origines sociales de ce personnel, rentré dans l'institution par la «petite porte». 1234 questionnaires, 22 entretiens et un certain nombre de note d'observations ont été analysés afin de développer une discussion critique sur les conditions de travail et de vie de ce personnel. Nous explorons comparativement également à la fois les expériences et les représentations de ces jeunes qualifiés, en situation de «précarité » de l'emploi, provenant de différents pays européens. Les inégalités spatiales et sociales à l'intérieur de cette « élite » transnationale européenne sont le fil conducteur de nos analyses.

Mots-clés: Commission européenne, diplômés, mobilité, Europe, emplois atypiques, inégalités
\end{abstract}

[Abstract] The European Commission (EC) in Brussels is often seen as a European-minded "body" of privileged civil servants. However, at the heart of this institution today, different types of workers exist, under various professional statuses. While looking closer at the social morphology, the diversity of backgrounds, and at hybridisation within the EC, it is clear that there is a certain gap between the positions of permanent and non-permanent workers. In this paper, we will focus on this population of skilled workers. We will try to depict the migratory paths, and social backgrounds of those that are said to be entering the institution through the "small door". 1234 questionnaires, 22 interviews, and a certain number of observations and notes have been analysed in order to develop a critical discussion on the EC staff conditions of work and life. We will then explore comparatively, both the experiences and representations of this skilled yet "precarious" youth, coming

\footnotetext{
${ }^{1}$ Aix Marseille Univ, CNRS, LAMES, Aix-en-Provence, France.

${ }^{2}$ Aix Marseille Univ, CNRS, LAMES, Aix-en-Provence, France.
} 
from different European countries. The spatial and social inequalities at the centre of this European transnational "elite" are at the core of our analysis.

Keywords: European Commission, graduates, mobility, Europe, atypical employment, inequalities

\section{Introduction}

L'emploi stable à la Commission européenne (CE) de Bruxelles serait-il lié à ses marges? Les études sur les institutions européennes traitent assez peu de la précarisation des parcours sur le segment secondaire de l'emploi à la CE de Bruxelles. Il s'agit dans cet article d'étudier des formes d'intégration professionnelle qui ont profondément évolué, non pas à partir de la situation de franges de la population peu qualifiée, mais à partir d'une population diplômée, a priori « favorisée », car le champ de «l'Eurocratie» n'est pas uniquement composé d'une jeune élite européenne cosmopolite et polyglotte, au parcours linéaire (Ballatore, 2013). Les situations précaires se développent dans ce secteur public européen qui passe pour incarner la stabilité de l'emploi (Georgakakis, 2012).

Des travaux classiques sur le monde ouvrier ont observé la façon dont la dualisation des statuts d'emploi tendait à se refléter dans la distribution de dispositions telles que l'aptitude à la mobilité et/ou l'esprit de compétition (Beaud, Pialoux, 1999). Qu'en est-il en ce qui concerne les agents des institutions européennes ? Pour répondre à cette question, nous nous focaliserons sur les profils des acteurs du segment secondaire ainsi que sur les effets de l'insécurité juridique sur leur vécu (Piore, Doeringer, 1971) de l'emploi qualifié à Bruxelles. Pour ce faire, nous avons enquêté auprès d'agents, d'employés et de stagiaires ayant un statut de cadre et/ou un niveau d'études égal ou supérieur au niveau 6 du cadre européen des certifications (CEC), qui font figure d'exemples dans les discours contemporains sur la mobilité et les économies dites « de la connaissance » (Berrebi-Hoffmann, 2012

Dans une première partie, nous nous pencherons sur les origines sociales, les profils et l'hétérogénéité des modes d'adaptation aux variétés des formes précaires d'emploi à la CE de Bruxelles pour ensuite, dans une seconde partie, tester l'hypothèse de l'avènement d'une main-d'œuvre qualifiée, mobile et flexible, qui connaît des situations de «précarisation » diverses et cumulatives.

\section{Encadré méthodologique}

L'enquête a été en partie effectuée par observation ethnographique, consignée dans un cahier de bord. Des entretiens semi-directifs ont également été menés en 2012 et 2013 auprès de jeunes actuellement ou anciennement en emploi à Bruxelles, stagiaires ou contractuels de la Commission européenne. Nous avons fait une analyse de contenu de 22 entretiens de jeunes de différentes nationalités (12 femmes et 10 hommes). Les Français, Italiens et Espagnols sont les plus 
représentés. On compte aussi une Portugaise (en couple avec un Italien), une Irlandaise, une Suédoise (en couple avec une Française) et une Grecque. Deux entretiens sont des entretiens de couples (un couple d'Italiens et un couple de femmes française et australienne). Certains interviewés ont été successivement stagiaires et/ou intérimaires, puis contractuels; d'autres simplement contractuels ou stagiaires, et deux sont des experts nationaux détachés (une Espagnole et un Italien). Nous avons aussi rencontré et interrogé des responsables syndicaux de la $C E$, ainsi que des responsables de la DGRH (Direction générale des ressources humaines).

Parallèlement à la réalisation des entretiens, nous avons aussi construit et fait circuler au début de l'année 2013 un questionnaire sur les mailing listes de la Commission (passant par l'intermédiaire des syndicats européens et en utilisant également les réseaux sociaux), auprès des fonctionnaires, experts nationaux, contractuels, intérimaires et stagiaires en emploi à la Commission européenne en 2012-2013. Ce questionnaire servait à dresser les profils sociaux et les parcours de manière comparative et, partant, permettait d'étudier le lien entre mobilité et cycles de vie, cette enquête reposant sur l'hypothèse qu'en fonction de la position des individus dans le cycle de vie, la mobilité n'a pas seulement un coût différent, elle a aussi un usage différent. La démarche comparative par l'intermédiaire de l'analyse quantitative permet d'aborder les questions au cœur de notre objet sous un angle différent, mettant à jour des dynamiques transversales, plus généralisables. Ainsi, 1234 individus ont répondu au questionnaire. Nous avons traité les données récoltées à l'aide du logiciel SPSS, puis du logiciel R, notamment pour les regroupements de modalités des variables.

\section{Origines et trajectoires scolaires : quelles corrélations avec les statuts d'emploi ?}

\subsection{Des agents en emploi temporaire qui cumulent de nombreux " capitaux »}

Selon les chiffres publiés par le budget général de l’Union européenne pour l'exercice 2009 et 2010, le personnel communautaire, tous statuts confondus, est passé de 280 agents en 1953 à environ 11000 en 1967 et à un peu plus de 37000 aujourd'hui (dont presque $70 \%$ travaillent à la CE située à Bruxelles). Parmi les membres du personnel temporaire enregistrés, environ $62 \%$ sont des agents contractuels. Cette catégorie regroupe encore aujourd'hui environ $20 \%$ de l'ensemble du personnel de la CE. D'autres catégories de salariés viennent grossir les rangs des agents qui ne sont pas fonctionnaires. Les statistiques de la $\mathrm{DGRH}^{3}$ ne comptabilisent pas les intérimaires mais cela cache mal une externalisation importante de certains services (notamment

\footnotetext{
${ }^{3}$ Statistics on European Commission Staff. URL : https://ec.europa.eu/info/about-europeancommission/organisational-structure/commission-staff_en.
} 
informatiques), qui fait disparaître un grand nombre de salariés, par exemple ceux travaillant à la commission des statistiques. Être permanent à la CE reste néanmoins la norme : c'est le fait de plus d'un agent sur deux.

Au moment de l'enquête, toutes les nationalités n'étaient pas représentées de manière égale parmi les fonctionnaires et chez le personnel en emploi atypique. Parmi les fonctionnaires, certaines nationalités étaient -et sont encoresurreprésentées dans les positions hautes d'assistants et d'administrateurs : les Belges, Allemands, Français, Italiens, Espagnols (par ordre décroissant). En ce qui concerne le personnel « temporaire » dont nous allons décrire dans le point suivant les statuts, les Belges restent en première position, mais les Italiens passent devant les Français, et les Espagnols devant les Allemands, en seconde et troisième positions parmi les nationalités les plus représentées. Les Grecs sont également nombreux à occuper des emplois atypiques, et les Portugais dépassent les Hollandais. Si on exclut les experts nationaux (qui sont certes «temporaires" à la Commission, mais pas dans une situation de précarité d'emploi, puisque fonctionnaires dans leur pays d'origine) l'Europe du Sud, en somme, est largement surreprésentée dans les emplois atypiques par rapport aux emplois permanents.

Tableau 1 - Statut d'emploi des agents travaillant à la CE de Bruxelles en 2013

\begin{tabular}{cccc}
\hline Statut de l'interviewé & Fréquence & $\%$ & Pourcentage cumulé \\
\hline Fonctionnaires & 642 & 52 & 52 \\
Intérimaires & 53 & 4,3 & 56,3 \\
Stagiaires & 55 & 4,5 & 60,8 \\
Contractuels & 311 & 25,2 & 86 \\
Agents temporaires & 56 & 4,5 & 90,5 \\
Experts nationaux & 37 & 3 & 93,5 \\
JRC, autres employés de & 12 & 1 & 94,5 \\
la CE & 68 & 5.5 & 100 \\
Ne travaillant pas & & 100 & \\
actuellement à la CE & 1234 & Source $:$ questionnaire passé en 2012-2013
\end{tabular}

L'origine sociale des agents des institutions européennes varie significativement en fonction des statuts d'emploił. Parmi les fonctionnaires et les contractuels interrogés

\footnotetext{
${ }^{4}$ Il existe deux grandes catégories de fonctionnaires à la CE : les administrateurs (AD) et les assistants (AST). Ils sont sélectionnés au moyen de concours généraux organisés par I'Office européen de sélection du personnel (EPSO). Les experts nationaux sont quant à eux
} 
par questionnaire, respectivement $38,9 \%$ et $39,9 \%$ ont un père appartenant à la catégorie socioprofessionnelle de "managers", chefs d'entreprises et professions intellectuelles supérieures, alors que presqu'un stagiaire et un expert national sur deux se trouvent dans cette situation. La profession de la mère est davantage significative, aussi est-ce celle que nous avons retenue ci-dessous dans le tableau 2. Ces écarts d'origine sociale en fonction du statut des interviewés peuvent être dus, en partie, à des écarts générationnels mais pas uniquement étant donné que les contractuels ont pour $15,4 \%$ d'entre eux une mère appartenant à cette catégorie supérieure, contre 43,6\% des stagiaires. Quel que soit le statut, les origines populaires sont rares: jamais plus de $6 \%$ des interviewés de chaque catégorie n'ont indiqué avoir ou avoir eu un parent ouvrier ou employé peu ou pas qualifié.

Tableau 2 - Origine sociale des agents travaillant à la CE de Bruxelles en 2013, en fonction du statut d'emploi (en \%)

\begin{tabular}{|c|c|c|c|c|c|c|}
\hline \multirow[b]{2}{*}{$\begin{array}{l}\text { Profession de la } \\
\text { mère }\end{array}$} & \multicolumn{6}{|c|}{ Statut de l'interviewé* } \\
\hline & Fonctionnaires & Intérimaires & Stagiaires & Contractuels & $\begin{array}{l}\text { Agents } \\
\text { temporaires }\end{array}$ & Ensemble* \\
\hline $\begin{array}{l}\text { Cadres et } \\
\text { professions } \\
\text { intellectuelles } \\
\text { supérieures }\end{array}$ & 15,4 & 22,6 & 43,6 & 18,3 & 19,6 & 18,2 \\
\hline $\begin{array}{l}\text { Professions } \\
\text { intermédiaires }\end{array}$ & 26 & 22,6 & 16,4 & 26,7 & 25 & 25,5 \\
\hline $\begin{array}{l}\text { Artisans, } \\
\text { commerçants et } \\
\text { chefs d'entreprise }\end{array}$ & 3 & 0 & 1,8 & 2,6 & 5,4 & 2,8 \\
\hline $\begin{array}{l}\text { Ouvriers, } \\
\text { employés peu ou } \\
\text { pas qualifiés, } \\
\text { personnel de } \\
\text { service }\end{array}$ & 5 & 5,7 & 3,6 & 4,8 & 3,6 & 4,8 \\
\hline H/F à la maison & 12,6 & 13,2 & 5,5 & 10,6 & 10,7 & 11,6 \\
\hline $\begin{array}{l}\text { Autres, retraités, } \\
\text { décédés }\end{array}$ & 38 & 35,8 & 29,1 & 37 & 35,7 & 37,1 \\
\hline Total & 100 & 100 & 100 & 100 & 100 & 100 \\
\hline$N=$ & 642 & 53 & 55 & 311 & 56 & 1117 \\
\hline \multicolumn{7}{|l|}{$\chi^{2}=32.6$} \\
\hline \multicolumn{7}{|l|}{$p<0,05$} \\
\hline \multicolumn{7}{|c|}{ *Sont exclus les experts nationaux, les JRC et autres employés de la $C E$, et les personnes ne travaillant pas à la $C E$} \\
\hline \multicolumn{7}{|c|}{$\begin{array}{l}\text { Lecture : } 15,4 \% \text { des fonctionnaires interviewés ont leur mère appartenant à la catégorie socioprofessionnelle « Cadres et } \\
\text { professions intellectuelles supérieures ». }\end{array}$} \\
\hline
\end{tabular}

détachés; ils appartiennent généralement à la fonction publique des États membres de I'UE. II existe aussi deux sous-catégories d'agents contractuels qui sont également recrutés par concours mais néanmoins engagés à durée déterminée, et deux types de stages à la CE (le stage administratif et le stage de traduction). 
Bien que les jeunes en contrat à durée déterminée de la CE ne soient pas tous issus $\mathrm{du}$ sommet de l'édifice social (fils et filles d'avocats d'affaires, de consultants internationaux, de cadres de la finance et management, etc.), les stagiaires et contractuels cumulent généralement de nombreux "capitaux», notamment économiques, mais aussi migratoires. Les jeunes des pays du Sud, comme le montre le tableau 3, sont d'origines sociales plus modestes et plus nombreux à appartenir à une catégorie, que l'on pourrait qualifier de moyenne inférieure ( lower middle class»). Nos entretiens montrent, nous le verrons, que le choix de mobilité intra-national ou intra-européen va agir pour eux comme un révélateur et les pousser à de nouvelles migrations et/ou à vouloir débuter une carrière à l'étranger.

Tableau 3 - Origine géographique des agents travaillant à la CE de Bruxelles en 2013, en fonction du statut d'emploi (en \%)

\begin{tabular}{|c|c|c|c|c|c|c|}
\hline \multirow{2}{*}{$\begin{array}{l}\text { Origine } \\
\text { géographique** }\end{array}$} & \multicolumn{6}{|c|}{ Statut de l'interviewé* } \\
\hline & Fonctionnaires & Intérimaires & Stagiaires & Contractuels & $\begin{array}{c}\text { Agents } \\
\text { temporaires }\end{array}$ & Ensemble* \\
\hline Europe de l'ouest & 50,2 & 49,1 & 36,4 & 41,5 & 48,2 & 46,9 \\
\hline dont Belgique & 21,2 & 18,9 & 1,8 & 19 & 7,1 & 18,8 \\
\hline \multirow{2}{*}{$\begin{array}{l}\text { dont France } \\
\text { dont Royaume- }\end{array}$} & 11,7 & 9,4 & 9,1 & 12,2 & 21,4 & 12,1 \\
\hline & & & & & & \\
\hline Uni & 4 & 7,5 & 7,3 & 1,6 & 7,1 & 3,8 \\
\hline \multirow{3}{*}{$\begin{array}{l}\text { Europe du nord } \\
\text { dont pays } \\
\text { scandinaves }\end{array}$} & 8,9 & 5,7 & 1,8 & 4,8 & 10,7 & 7,3 \\
\hline & & & & & & \\
\hline & 6,7 & 1,9 & 1,8 & 3,2 & 3,6 & 5,1 \\
\hline Europe de l'est & 17,1 & 18,9 & 21,8 & 17,7 & 12,5 & 17,4 \\
\hline Europe du sud & 22,9 & 26,4 & 30,9 & 32,8 & 26,8 & 26,4 \\
\hline \multicolumn{7}{|l|}{$\begin{array}{l}\text { Autres, ou } \\
\text { plusieurs }\end{array}$} \\
\hline nationalités & 0,9 & 0 & 9,1 & 3,2 & 1,8 & 2,0 \\
\hline Total & 100,0 & 100,0 & 100,0 & 100,0 & 100,0 & 100,0 \\
\hline$N=$ & 642 & 53 & 55 & 311 & 56 & 1117 \\
\hline Non réponse $=$ & 0 & 0 & 0 & 0 & 0 & 0 \\
\hline \multicolumn{7}{|l|}{$\chi^{2}=43,7$} \\
\hline \multicolumn{7}{|l|}{$p<0,01$} \\
\hline \multicolumn{7}{|c|}{$\begin{array}{l}\text { *Sont exclus les experts nationaux, les JRC et autres employés de la CE, et les personnes ne travaillant pas à la CE } \\
\text { ** Europe de l'Ouest: Autriche, Allemagne, Belgique, France, Irlande, Luxembourg, Pays-Bas, Royaume-Uni }\end{array}$} \\
\hline \multicolumn{7}{|c|}{ Europe du Sud: Grèce, Italie, Malte, Portugal, Slovénie, Espagne, Chypre } \\
\hline \multicolumn{7}{|c|}{ Europe de l'Est: Bulgarie, Hongrie, Pologne, République Tchèque, Roumanie, Slovaquie } \\
\hline \multicolumn{7}{|c|}{ Europe du Nord: Estonie, Lettonie, Lituanie, et pays scandinaves (Finlande, Suède, Danemark) } \\
\hline \multicolumn{7}{|c|}{ Lecture : 50,2 \% des fonctionnaires interviewés venaient d'un pays d'Europe de l'Ouest. } \\
\hline
\end{tabular}

\subsection{Des profils d'étude spécifiques aux parcours atypiques}

Notre enquête quantitative montre par ailleurs qu'il existe bien des profils d'études, des filières particulières qui mènent à la CE de Bruxelles, et ce, d'autant plus nettement que les interviewés sont jeunes et stagiaires. Ainsi, parmi l'ensemble des fonctionnaires, intérimaires, stagiaires, contractuels, et agents temporaires, $35,4 \%$ des interviewés ont indiqué avoir suivi des études de droit et/ou de sciences 
politiques, et $18 \%$ ont suivi des études d'économie. Ces pourcentages varient en fonction des statuts d'emploi. Ainsi, 41,6\% des contractuels ont indiqué avoir suivi des études de droit et/ou de sciences politiques et 15,9\% des études d'économie; $21,1 \%$ des fonctionnaires avaient suivi des études d'économie. Les stagiaires étaient, par contre, 67,3\% à avoir suivi des études de droit et de sciences politiques.

Les intérimaires, les agents temporaires (non recrutés sur concours) ainsi que les experts nationaux sont ceux qui présentent la diversité des filières d'études la plus grande, avec une part beaucoup plus importante de diplômés de sciences dites « exactes ». Les diplômés en sciences humaines sont les moins bien représentés. Il existe de plus en plus de travaux qui apportent des éléments de connaissance sur les dispositions favorables à une carrière dans les métiers de l'Europe (Michon, 2004, 2008 ; Michel, Robert, 2010 ; Georgakakis, 2012, 2013). Ils renseignent sur certains processus d'acquisition, ainsi que sur certains types de formations et d'institutions nationales ou supranationales, notamment la plus connue et spécialisée d'entre elles dans le milieu européen : le Collège d'Europe à Bruges (Schnabel, 1998). Ils laissent cependant souvent de côté le personnel temporaire et les différences de parcours suivant les appartenances géographiques et sociales. L'enthousiasme affiché et le parcours linéaire de certains stagiaires vers le fonctionnariat européen cache, comme nous allons le voir dans cette seconde partie, une précarisation grandissante des parcours professionnels d'autres diplômés aux trajectoires plus chaotiques.

\section{Variété des formes précaires d'emploi et « servitude volontaire "}

\subsection{Un espace d'incertitudes non éprouvé comme « précaire »}

Dans cette partie, nous allons nous intéresser à l'insertion professionnelle d'une fraction de la population interrogée dont la trajectoire est nettement moins linéaire (stages non rémunérés, périodes de chômages, etc.) que celle des jeunes d'origine sociale "très élevée » dotés de parcours brillants et formés dans des institutions d'enseignement élitistes. Javier, dont le père était fonctionnaire communal et la mère gérante d'un bar, qui travaillait pendant ses études de journalisme à Madrid, dit ainsi avoir "perdu du temps » en raison d'un manque d'information sur les possibilités de travail à l'étranger. En effet, après un séjour Erasmus, il décide de poursuivre et finir ses études en Allemagne mais ce n'est que suite à plusieurs stages non rémunérés, notamment en Croatie à l'OCDE, afin « d'enrichir son Curriculum Vitae », qu'il décide de tenter les concours à la CE sur les conseils de collègues.

Agent contractuel au moment de l'enquête, Javier avait été engagé pour trois ans. Les tests de sélection qu'il avait passés étaient les mêmes que pour les fonctionnaires, en dehors du test oral qui n'existe pas. Le procédé est, selon les documents officiels, «quicker» et «lighter». Une fois ses trois années à la CE terminées, nous avons 
recontacté Javier, pour connaître sa trajectoire. N’ayant pas réussi le concours de fonctionnaire qu'il a tenté de nombreuses fois, il cherchait par tous les moyens à être embauché dans les agences, dans certaines organisations non-gouvernementales ou comme lobbyiste. Il rejoint ainsi la multitude des candidats qui gravitent autour des institutions européennes. Le mode de recrutement entretient leur incertitude. En effet, les candidats qui obtiennent des résultats supérieurs à un certain seuil au concours de contractuel sont placés sur une liste de réserve. Il n'existe en fait aucune garantie d'être recruté, même si les résultats sont satisfaisants, car la Commission examine la liste des candidats qui ont réussi et prépare des «short-lists». Des entretiens ont aussi souvent lieu. Même une fois recruté, il faut s'assurer une continuité d'emploi. Andrea un jeune italien interrogé, décrit son parcours une fois recruté pour remplacer une personne en congé maternité, un contexte d'incertitudes multiples, une sorte de "course d'obstacles» qui lui a pris beaucoup de temps et d'énergie pour assurer une certaine continuité d'emploi durant ces trois ans :

Ils m'ont pris pour remplacer une fonctionnaire en congé maternité, j'ai eu un contrat pour un an [...] il y a deux types de contrat, il y a le poste où tu prends la place d'un autre agent contractuel qui a fini ses trois ans, mais même dans ce cas ils te font qu'un contrat d'un an, renouvelable! Après une autre a pris un congé toujours dans la même unité [...] donc ils m'ont renouvelé pour huit mois, mais seulement pour huit mois, incroyable ! [...] Et après ces huit mois, ils ne pouvaient plus me renouveler, plus de poste ! [...] Ce qui est absurde à la Commission est que tout est divisé en catégories, dans le sens où beaucoup de personnes sont parties de mon unité, mais si un fonctionnaire part, ils ne peuvent pas te dire, tu connais le travail, tu prends son poste, non, ils prennent un fonctionnaire qui connaît rien à rien! [...] après ces huit mois, plus de budget alors j'ai commencé à envoyer des $\mathrm{CV}$, pour changer d'unité, et là pas toujours facile, mais j'avais trouvé un poste dans une autre unité, mais en janvier, alors que mon contrat finissait en mai, en avril ils me disent, c'est pas sûr [...] tu te rends compte ? [...] et ça a continué comme ça, où ils me disaient ni oui, ni non [...] alors je leur ai dit, ok je fais trois mois de vacances, si vous me dites qu'en septembre vous me prenez à $100 \%$ ! [...] Bon, c'est un peu pénible, mais il y a l'employment bank [...] tu fais la procédure belge, mais c'est la Commission qui paie, mais dans l'incertitude, j'ai cherché encore un autre poste et j'ai trouvé celui-ci dans cette DG [...] je devais commencer au premier juin et genre le 24 mai pour le 25 , ils m'ont dit, on te prend! (Andrea, nationalité italienne, 31 ans. Études: économie industrielle. Statut : contractuel. Père et mère : professeurs de collège)

Les profils scolaires des intérimaires que nous avons interrogés sont variés, car la Commission les engage par le biais d'agences d'intérim. La mission ne doit pas 
dépasser six mois. En outre, des services sont externalisés, dont une partie des services informatiques. Ils n'entrent donc pas dans les statistiques des travailleurs temporaires établies par la CE. Nous en avons rencontré, diplômés des universités, qui nous ont décrit leurs conditions de travail en bas des immenses immeubles de verre et qui sont fréquemment rémunérés bien en-dessous du revenu moyen des contractuels. La question des bureaux est importante et cristallise des hiérarchies et des différences, des inégalités de rétribution et de considération.

Chez les intérimaires, le chômage est en quelque sorte "intégré » au système de l'emploi et généralise l'insécurité professionnelle, avec peu d'avantages et beaucoup d'inconvénients qui sont étrangement intriqués. Le fait de pouvoir et de devoir, après six mois de contrat, rentrer chez soi ou partir en congé un mois, avant éventuellement d'être réembauché, fait partie de ces avantages évoqués (plaisir de retrouver famille et amis dans son pays d'origine ou de pouvoir voyager), mais aussi des inconvénients liés aux incertitudes existentielles, car la réembauche par la suite n'est pas garantie. De plus, souvent, ces jeunes n'ont pas travaillé suffisamment pour pouvoir bénéficier de droits au chômage. Pourtant, comme Paolo qui a émigré pour palier un échec scolaire relatif, les jeunes intérimaires affichent sans cesse leurs performances, tout en étant conscients de se mouvoir dans un système individualisé, en espérant toujours en tirer profit :

Je suis resté intérimaire très longtemps, trop, c'est fatigant ! [...] j'ai fait des boulots d'exécution plutôt, mais pendant ce temps, je me suis dit faut que tu trouves autre chose, donc je passais beaucoup de temps à écrire à la responsable du programme qui m'intéressait [...] je l'ai littéralement harcelée! Une fois elle m'a répondu ça va on a compris que tu es intéressé, mais ça c'est du harcèlement! Parce que tous les lundis je lui envoyais mon CV. Et je lui ai dit que ok, j'arrêterais de la harceler le jour où ils m'appelleront pour travailler [rire]. Et ça s'est passé comme ça ! [...] Du coup j'ai fait pendant des années un peu tout et n'importe quoi [...] le travail d'intérim, c'est pas facile d'autant qu'il y a des intérimaires de catégorie $A$, et $B$ et moi j'étais dans la catégorie $B$, au sens où ils me faisaient un renouvellement chaque mois et parfois il $y$ avait aussi des mois off .... Alors que les catégories A souvent ont des contrats de six mois et donc ont plus de visibilité. (Paolo, intérimaire, nationalité italienne, 38 ans. Études de comptabilité. Père: avocat. Mère : assistante sociale)

Parmi les contractuels et les intérimaires, nous avons observé une multitude de situations complexes dans lesquelles les individus cherchent un compromis entre des aspects de leur travail qui leur permettent de se valoriser, et d'autres qu'ils acceptent plus ou moins par contrainte, parfois en les rationalisant, pour mieux les supporter. C'est le cas de Sabine : 
J'ai fini mon stage en juillet 2009, j'ai galéré ensuite jusqu'en octobre ou novembre 2009, et là, j'ai trouvé un stage à la DG [X] à l'époque, en intérim... [...] c'était avec une agence, donc c'est le truc, tu sais toutes les semaines, tu fais remplir ton truc, oui, j'ai bien travaillé comme une grande et tu fais remplir à ton chef, c'est assez infantilisant, hein ? Mais du coup, c'était quand même du fric et du boulot et voilà. C'était un peu chiant, c'était un travail administratif et... ce n'était pas du secrétariat, mais c'était de l'administratif chiant au début, parce que je remplaçais quelqu'un, mais qui ne revenait pas, donc je restais, je restais, je restais et j'ai fini par rester six mois là. [...] en 2010, à ce moment-là, ça a changé [de direction], je pensais que j'allais faire plein de trucs intéressants et puis, je me suis rendue compte, que finalement non. (Sabine, nationalité franco-marocaine, 27 ans. Études: IEP. Mère: professeure de biologie. Père : pharmacien)

Ainsi, la précarisation ne se réduit pas au statut d'emploi (temporaire, peu protégé, etc.) mais s'étend à la dimension subjective, relationnelle et existentielle qui est liée à cette activité. Au-delà de l'instabilité croissante ressentie, la population enquêtée semble touchée par une augmentation des mobilités contraintes au sein d'un microcosme bruxellois de plus en plus segmenté en fractions spécifiques (Ramaux, 2006). Les diplômés de l'enseignement supérieur qui sont issus des familles les moins favorisées sont d'autant plus affectés par le processus de précarisation dans leurs parcours, du fait de l'absence d'une base de solidarité privée fondée sur le patrimoine familial et de réseaux professionnels peu importants. Ils ne se reconnaissaient cependant pas nécessairement comme "précaires» pour autant. En effet, pour de nombreux jeunes que nous avons rencontrés, être précaire, c'est avoir un "bad job » dans leurs pays d'origine respectifs (Green, Mostafa, Parent-Thirion et al., 2013) et non pas vivre dans l'insécurité de l'emploi à Bruxelles, à condition que la rémunération est bonne.

\subsection{Socialiser pour être recruté ?}

Le nombre de demandes pour les stages a été grandissant ces dernières années ${ }^{5}$. Environ $30 \%$ des postulants se retrouvent dans ce que la Commission appelle le « Blue Book» et devront ensuite, soit attendre d'être contactés par une DG, soit « faire du lobbying » pour être recrutés. Un quota par nationalité existe. En mars 2012 par exemple, le bureau des stages a enregistré 8778 candidatures en ligne. Parmi elles, 2314 candidats étaient italiens, 724 espagnols et 536 grecs, alors que seulement 404 Allemands et 281 jeunes britanniques candidataient. Seuls $4 \%$ des Italiens ont trouvé une place à l'issue du processus, contre $15 \%$ des Britanniques. Il importe ici de

\footnotetext{
${ }^{5}$ Le niveau minimum d'études requis est la licence, et la connaissance d'au moins deux langues de l'UE est exigée. Pour candidater il n'y a plus de limite d'âge.
} 
préciser que le traitement mensuel est d'environ 1000 euros, dans un contexte où dans certaines régions d'Europe les stagiaires ne reçoivent bien souvent aucune rémunération ou un simple remboursement de frais occasionnés par l'activité. Dans ce contexte, ces stages sont très convoités, une forte sélection existe et les profils des candidats reçus tendent à se ressembler. Ils sont nombreux par exemple à être passés par le collège d'Europe de Bruges, comme Laure :

Je me suis spécialisée en relations internationales, et notre dernière année de master [à Sciences Po Lille] la $5^{\text {ème }}$ année, on peut se spécialiser encore plus, et là donc j'ai fait analyse des conflits et construction de la paix. Et ça m'a servi pour entrer au collège d'Europe de Bruges [...] la sélection est faite par le Ministère des Affaires étrangères français [...] quand tu arrives à Bruges, tu te rends compte que tous les français ont fait Sciences Po [...] la Commission fait une présélection, et quelques mois après tu sais que tu as été présélectionné et à ce moment-là les différents services de la Commission choisissent leurs stagiaires dedans. [... ] Sur ma session de stage, on est une cinquantaine quand même, sur une promo de 300 à Bruges, donc c'est quand même pas mal, il y en a dans tous les services. (Laure, stagiaire, nationalité française, 24 ans. Études: IEP de Lille/Collège d'Europe. Parents : cadres supérieurs internationaux (père dans le pétrole; mère dans la banque)

Tableau 4 - Programmes d'échanges auxquels ont participé les agents travaillant à la CE de Bruxelles en 2013, en fonction du statut d'emploi (en \%)

\begin{tabular}{|c|c|c|c|c|c|}
\hline \multirow[b]{2}{*}{$\begin{array}{l}\text { Participation à un } \\
\text { programme } \\
\text { d'échange }\end{array}$} & \multicolumn{5}{|c|}{ Statut de l'interviewé* } \\
\hline & Fonctionnaires & Intérimaires & Stagiaires & Contractuels & Ensemble* \\
\hline $\begin{array}{l}\text { Un seul } \\
\text { programme } \\
\text { dont Erasmus }\end{array}$ & 22,1 & 20,0 & 53,1 & 33,1 & 27 \\
\hline $\begin{array}{l}\text { uniquement } \\
\text { dont programme }\end{array}$ & 12,7 & 8,6 & 38,8 & 22,8 & 17 \\
\hline hors Erasmus & 9,4 & 11,4 & 14,3 & 10,3 & 10 \\
\hline $\begin{array}{l}\text { Plusieurs } \\
\text { programmes dont }\end{array}$ & 29 & 86 & 204 & 72 & 54 \\
\hline Aucun programme & 75 & 71,4 & 26,5 & 59,7 & 67,6 \\
\hline Total & 100 & 100 & 100 & 100 & 100 \\
\hline$N=$ & 543 & 35 & 49 & 263 & 890 \\
\hline Non réponse $=$ & 99 & 18 & 6 & 48 & 171 \\
\hline
\end{tabular}

*Sont exclus les experts nationaux, les JRC et autres employés de la $\mathrm{CE}$, les personnes ne travaillant pas à la CE.

Lecture : 2,9\% des fonctionnaires interrogés ont déjà fait un séjour Erasmus en plus d'un séjour dans un autre programme d'échanges. 
Les stagiaires de la CE voient cette expérience comme une étape vers une carrière européenne ou internationale; ils évoquent rarement la fatigue et le cumul des emplois atypiques que d'autres salariés "temporaires", plus âgés, disent subir. Leur travail, néanmoins, semble coloniser beaucoup plus la sphère privée que pour d'autres catégories de travailleurs en emploi temporaire à la Commission (Cingolani, 2012). Ces stagiaires acceptent une forme de "servitude volontaire» (Durand, 2004; Salecl, 2011) temporaire dans l'espoir souvent (mais aussi dans l'incertitude) d'être un jour recrutés comme fonctionnaires. Au cours de cette période, le travail à mener sur soi pour renforcer les chances d'un recrutement futur les mobilise souvent au point de brouiller la dichotomie normalement observable chez les salariés entre travail et non-travail. Il ressort des entretiens que leur temps «libre » est souvent occupé par des "loisirs» qui permettent une formation permanente (cours de langues, par exemple) ou par la création de réseaux qui pourront leur être utiles pour la poursuite de leur carrière.

Les stagiaires sont aussi fortement incités, par l'intermédiaire d'un comité des stages institutionnalisés, à faire des activités et sorties extra-professionnelles ensemble. Du fait de ces incitations à socialiser, ceux ayant déjà l'expérience d'un séjour Erasmus y trouvent fréquemment des ressemblances avec leur « expérience » à la CE.

L'opacité en termes de sélection des stagiaires est évoquée par quelques recruteurs mais surtout par les recrutés et ceux qui ne l'ont pas été. Il y a bien, à la lecture des entretiens et des questions ouvertes de notre questionnaire, des "héritiers » du monde clos bruxellois et d'autres qui n'en sont pas, et certains soupçonnent que cette distinction a pu jouer dans la sélection. Pour la première sélection sur dossier, il existe des critères clairs (niveau d'études, maîtrise des langues par exemple) mais, une fois sur le «blue book», les stagiaires et les fonctionnaires en charge du recrutement ne sont souvent pas en mesure de dire pourquoi certains ont été écartés alors que d'autres ont été retenus. Néanmoins, tous parlent de l'importance de faire du "lobbying", de "se vendre», d'envoyer des emails à des responsables d'unités. Certains évoquent également le fait d'être passés par l'intermédiaire d'un professeur, d'un ami ou d'un collègue qui connaissaient directement une ou plusieurs personnes en poste dans une Direction générale, comme Patrick:

Vers mars, j'ai commencé à envoyer des emails à quelques personnes, mais pas beaucoup, assez ciblées, [...], dans les unités dans lesquelles je voulais travailler [...] puis j'ai une amie, en fait, qui a fini son contrat, là où je suis maintenant et donc, en discutant, elle m'a dit, ah, mais moi je finis dans deux mois, donc si tu veux je donne ton CV et euh... [...] J'ai fait un entretien, j'ai juste eu un entretien et ils m'ont dit, c'est bon. (Patrick, nationalité belge, 30 ans. Études: sciences politiques. Père: avocat. Mère : présidente d'un IEE. Marié, 1 enfant) 
Conformément aux injonctions adressées aux stagiaires, s'appuyer sur le horstravail pour nouer des relations avec ses collègues semble un atout en vue du recrutement. Du fait de l'impératif de socialisation qui en résulte, « [l]es cadres de la socialisation européenne » dépassent les seuls lieux de travail pour s'étendre à la plupart des domaines de la vie privée des stagiaires (Michel, Robert, 2010 : 15).

\subsection{Bruxelles : passage ou aboutissement d'un parcours de mobilité?}

Pour de nombreux jeunes originaires du sud de l'Europe, à l'instar de Raquel, une stagiaire portugaise qui a étudié le droit et qui est restée quelques années à Bruxelles après son stage avant de rentrer au Portugal, les emplois non-permanents au cœur ou autour des institutions européennes sont des emplois d'attente ou encore des choix provisoires, qui se sont dessinés faute de mieux dans le pays d'origine.

Vivre et travailler à Bruxelles résulte d'une opportunité particulière et plus contingente chez des diplômés du sud de l'Europe qui n'ont pas fait d'études de sciences politiques, de droit international ou d'études européennes, et sont à la recherche d'un travail à la hauteur de leurs qualifications. Même si les diplômés qui ont changé plusieurs fois de lieu d'étude et de travail, comme Stefano, italien passé notamment par l'ENS (École normale supérieure) et l'OCDE de Paris pour un stage, avant de travailler à la CE de Bruxelles, disent fréquemment ne pas avoir émigré, ne pas être venu à Bruxelles "par désespoir», ils soulignent aussi fréquemment l'incapacité de leur pays d'origine à intégrer les jeunes diplômés du supérieur universitaire :

De manière générale, je me vois mal trouver quelque chose qui m'incite

à rentrer en Italie... je suis parti content, tranquille, sans haine de l'Italie, mais avec le recul, maintenant que j'aimerais rentrer, y aura-t-il un poste pour moi ? Ben, je ne sais pas... plutôt pas! C'est ça le problème. (Stefano, nationalité italienne, 28 ans. Études: doctorat d'économie politique; Père: enseignant de lettres. Mère: conseillère financière dans une banque)

Nos interviewés issus de milieu favorisé recourent, en fonction de leurs moyens, à des solutions scolaires distinctives (choix du multilinguisme, écoles internationales, filières sélectives entre autres) ainsi qu'à des stratégies de mobilité géographique, pour maintenir et reconstruire tout un ensemble de frontières sociales, que la massification scolaire a eu tendance à effacer au fil des décennies.

\section{Conclusion}

La précarisation nous semble une notion pertinente pour décrire les parcours de nombreux diplômés qui possèdent un fort capital culturel et international mais aspirent à entrer dans des segments du marché du travail qualifié fortement 
concurrentiels (Draelants, Ballatore, 2014). Ce haut niveau de concurrence favorise la constitution d'un moratoire d'emploi précaire pour des individus destinés, en grande partie, à des carrières de cadres administratifs de l'international.

Cependant, notre enquête fait ressortir des différences notables parmi ces précaires temporaires entre, d'une part, ceux qui avaient opté pour "l'Europe» précocement, depuis le moment du choix même de leurs études et, d'autre part, ceux dont les parcours de mobilité se sont dessinés peu à peu, faute de pouvoir trouver dans leurs pays respectifs une place conforme à leurs attentes. La satisfaction en général affichée par les stagiaires de la CE masque une réalité plus mitigée et contrastée, faite de parcours sinueux pour certains jeunes qualifiés de régions européennes "périphériques » (du sud notamment), assignés au segment secondaire du marché du travail européen.

Notre enquête contribue ainsi à démystifier la figure héroïsée " du cadre nomade " souvent appliquée sans examen sur la réalité sociale (Colombi, 2016). Plus précisément, nous l'avons ici décantée en deux pôles qui s'en écartent, l'un par le haut et l'autre par le bas. Par le haut: les postes temporaires à Bruxelles interviennent au sein des parcours de jeunes fortement dotés scolairement et/ou socialement, dont la carrière s'est surtout construite par et dans les institutions européennes. Plutôt qu'un héroïsme de la mobilité, ce groupe illustre ce que l'on pourrait appeler paradoxalement une sédentarité de l'international. Par le bas : ces positions sont aussi investies par des jeunes qui risquent davantage, du fait de leurs trajectoires moins linéaires et déjà marquées par l'emploi précaire, d'y être maintenus, voire de subir le chômage. Ainsi, les mêmes formes d'emploi prennent des significations divergentes selon les origines sociales, scolaires et géographiques de ceux qui les occupent, cette dualisation de la précarité elle-même tendant à refléter les trajectoires sociales divergentes entre nations européennes.

\section{Bibliographie}

Ballatore M. (2010), Erasmus et la mobilité des jeunes européens, Paris, Presses universitaires de France.

BALlatoRE, M. (2013) «Travailler à la Commission Européenne de Bruxelles sans être fonctionnaire: un entre-deux confortable entre formation et emploi?», Chroniques du travail, $\mathrm{n}^{\circ}$ 3, p. 119-139.

Beaud S., Pialoux M. (1999), Retour sur la condition ouvrière. Enquête aux usines Peugeot de Sochaux-Montbéliard, Paris, Fayard.

BERREBI-HOFFMANN I. (2012), "Impossible subordination? Figure de la relation hiérarchique dans les services informatique», in P. CingolanI (dir.) Un travail sans limites? Subordination, tensions, résistances, Toulouse, Érès, p. 59-80. 
Cingolani P. (dir.) (2012), Un travail sans limites ? Subordination, tensions, résistances, Toulouse, Érès.

Colombi D. (2016), Les usages de la mondialisation: mobilité internationale et marchés du travail en France, thèse de doctorat, Sciences Po Paris.

DRAELANTS H., BALLATORE M. (2014), « Capital culturel et reproduction scolaire. Un bilan critique », Revue française de pédagogie, $\mathrm{n}^{\circ} 186$, p. 115-142.

DURAND J.-P. (2004), La chaîne invisible. Travailler aujourd'hui : flux tendu et servitude volontaire, Paris, Seuil.

GEORGAKAKIS D. (dir.) (2012), Le champ de l'Eurocratie. Une sociologie politique du personnel de l'UE, Paris, Économica.

GEORGAKAKIS D. (2013), «What Is Happening to the Staff of the European Institutions? A Cross-Disciplinary View», in B. REINALDA (dir.), Routledge Handbook of International Organization, Londres, Routledge, p. 287-298.

Green F., Mostafa T., Parent-Thirion A., Vermeylen G., Van Houten G., Biletta i. et Lyly-YRJANAINEN M., (2013), «Is Job Quality Becoming More Unequal?», Industrial and Labor Relations Review, n 66, p. 753-784.

Michel H., Robert C. (2010), La fabrique des «Européens ». Processus de socialisation et construction européenne, Paris, Presses universitaires de France.

Michon S. (2005), «Les assistants parlementaires des députés européens : étude d'un groupe d'auxiliaires au Parlement européen », in G. COURTY (dir.), Le travail de collaboration avec les élus, Paris, Michel Houdiard Éditeur, p. 118-135.

Michon S. (2008), «Assistant parlementaire au Parlement européen: un tremplin pour une carrière européenne », Sociologie du travail, vol. 50, nº 2, p. 169-183.

Piore M. J., DoERINGER P. (1971), Internal Labor Markets and Manpower Adjustment, New York, D.C. Heath and Company.

Ramaux C. (2006), Emploi : éloge de la stabilité. L'État social contre la flexicurité, Paris, Mille et une nuits.

SALECL R. (2011), The Tyranny of Choice, Londres, Profile Book.

SCHNABEL V. (1998), "Élites européennes en formation, les étudiants du collège de Bruges et leurs études », Politix, $n^{\circ}$ 43, p. 33-52. 